\title{
A System Dynamics Model for Risk Analysis during Project Construction Process
}

\author{
Jiangping Wan, Yaqiong Liu \\ School of Business Administration, South China University of Technology, Guangzhou, China \\ Email: csjpwan@scut.edu.cn, liu20090770212@163.com
}

Received 19 April 2014; revised 24 May 2014; accepted 1 June 2014

Copyright (C) 2014 by authors and Scientific Research Publishing Inc.

This work is licensed under the Creative Commons Attribution International License (CC BY). http://creativecommons.org/licenses/by/4.0/

\begin{abstract}
Risk analysis is an important part for many large and medium-sized construction projects; meanwhile the correct and reasonable project risk analysis can be conducive to risk management. Based on the results of the case study supplemented by a literature research, this paper presents an approach to develop a system dynamics model for risk analysis during project construction process. At first, this paper gives a brief description of risk factors project construction and system dynamics. The risks as well as the causal relationship diagram between them are determined in order to illustrate the risk interaction of the project construction with system dynamics model. A recapitulation of the findings and a prospect on future research conclude the paper.
\end{abstract}

\section{Keywords}

Project Construction, Risk Analysis, System Dynamics

\section{Introduction}

Project is one-time construction business with a special target, which is also under the condition of certain constraints, including limited time, resources, quality, etc. [1]. As the general large and medium-sized construction projects with complicated construction conditions, coordination difficulties, sensitive construction environment, high social awareness and other characteristic [2], so according to this characteristics, this paper analyzed potential risks and initially identified the schedule risk, quality of construction, cost risk, environmental risk, technology risk, design risk and benefit risk as the main risk affecting the project.

There are many sophisticated risk analysis method, the commonly used methods now are structural decomposition and expert investigation method. Structural decomposition method will decompose the project risk according to the law of the thing itself and the historical experience of individual, such as fault tree, decision trees, probability trees; the expert investigation method mainly includes brainstorming method, Delphi method, curtain 
scene analysis method, etc. [3]. Both these two methods analyze the various risk factors during project construction process comprehensively, but they didn't take the linkages between risk factors into account. The fact is the project construction risks are not only much more but also have an interaction between each other because of its complex engineering project construction conditions. System dynamics model can show that the interactions between risk variables using the feedback loop, simulate the development trend based on the information of the project current status, and analyze the importance of the various project risks quantitatively [4].

The risks as well as the causal relationship diagram between them are determined in order to illustrate the risk interaction of the project construction with system dynamics model in the following sections.

\section{Developing a System Dynamics Model}

\subsection{System Dynamics}

System dynamics models are mainly used to depict and analyze dynamic systems. These model is a computer simulation technique for the study of system dynamic behavior and originally developed at the Massachusetts Institute of Technology by Dr. Forrester; also he made a major breakthrough for the system dynamics in 1958 [5]. Dr. Forrester published "industrial dynamics" and "systems theory" works, laying the foundation of system dynamics [6]. Meantime, system dynamics models have been relatively mature and applied in mangy disciplines and in many contexts.

Establishing a causal relationship between the variables is the core of system dynamics model. Causality relationship loop diagram is used to analyze the complex interactions between the system's internal variables, which can have single or multiple feedback loops. Interrelated variables form a causal chain, meanwhile, the polarity of the causal chains can be enhanced also be weakened. The polarity between the causal chains between the differrent variables may be different; therefore, the nonlinear feedback loop of the whole system has been formed.

\subsection{Structure of the Causal Relationship Diagram Model}

The project risks are analyzed in the view of project builders. The schedule risk means the loss by schedule delay or direct loss caused by uncertainties in the predetermined period of time. Quality of construction risk is the quality problems which are unexpected and due to the various factors in the construction process. Cost risk refers to the dangers by increasing cost. Environmental risk means the degree of harm to the environment by sudden accident in the construction process. Technology risk refers to the construction barriers caused by inadequate levels of the technical personnel and construction technology. Design risk can be understood as the potential for negative and undesirable consequences occur during design work. Benefit risk the level of the project benefits meet the expected expectations. A causal relationship diagram on the base of the risks identified above is established, including the schedule risk, quality of construction, cost risk, environmental risk, technology risk, design risk and benefit risk. The causal relationship diagram is illustrated in Figure 1.

All the important risk variables during project construction are illustrated in Figure 1. The variables without box is state variable, in addition, all state variables combined are characterizing the current state of the complete system. The variables with gray box are auxiliary variables, which are introduced between the actions from the source to the decision, expressing feedback assisted decision. The blocked variables are input variables. These variables are not affected by other variables. The connection between the variables which describes the influence of variables, and the arrows indicate the direction of impact. Arrow polarity "+" means that the influence between two variable is proportional, “-” indicates inversely [7]. When evaluating whether the influence is proportional or inversely proportional, we have to assume that the other variables are constant. The causal relationship diagram for risk analysis of project construction process, including three feedback loops, is established in this paper.

\subsection{Variables of the System Dynamics Model}

According to the basic features of the project, we have chosen the schedule risk, quality of construction, cost risk, environmental risk, technology risk, design risk and benefit risk as the state variables of the system. Select auxiliary variables for each state variable. For instance, natural disaster, rework, planned duration, estimated total time are selected as the auxiliary variables for schedule risk; rework, wage variation, the change of budget are selected as auxiliary variables for cost risk; construction materials, production efficiency, construction tech- 


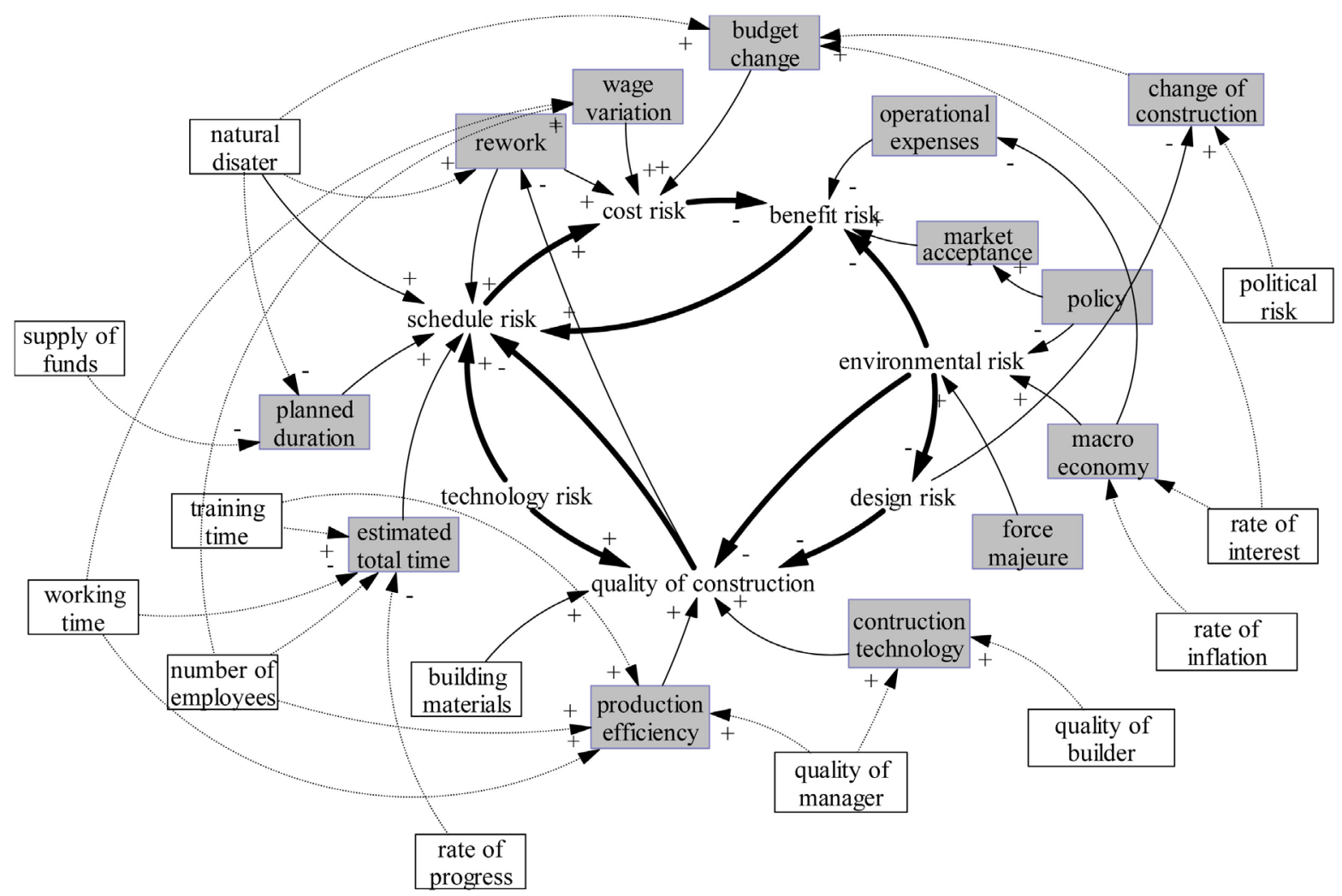

Figure 1. Causal relationship diagram for risk analysis of project construction. legend: cond loop, $--\rightarrow$ third loop, no box as state variable, grey box as auxiliary variable, colorless box as input variable.

nology are selected as auxiliary variables for the quality of construction; operational expenses, market acceptance are selected as auxiliary variables for benefit risk; policy, macro economics, force majeure are selected as auxiliary variable for environmental risks. Input variables are elicited from state variables and auxiliary variables, and we will describe them in detail below.

The schedule of a project construction is crucial. It, whether funds are adequate or not, will affect the planned schedule and thus affect the schedule risk. So the schedule risk will be reduced if adequate funds are guaranteed. Increasing the number of employees and working hours, improving the rate of progress of the construction project, and enhancing the support of completing the project on time, these will have a positive impact on reducing the risk [8]. The increase of natural disasters will increase the schedule risk. On the one hand, the increase in training time will improve the ability of employees, the production efficiency and the quality of construction, and it will reduce the schedule risk. Meanwhile, three input variables-the increase of working hours and the number of employees, and the improvement of managers' quality will improve the production efficiency and the construction quality [9]. The quality of construction will be affected directly by building materials, hence, it be improved by providing building materials with better quality. The quality of employees is another input variable which have an effect on the construction quality, for the construction technology can be improved by the improvement of quality of employees, so as to ensure the quality of construction.

Increase the number of employees and working hours can bring a lot of positive effects, but also brings a great deal of cost burden, increasing the cost risk. Natural disasters will increase rework, but also cause the economic losses and casualties, which would make the cost risk increased.

The input variables are the detailed description of the project risk. In addition, we need consider project risks from the macro level. The rate of inflation and interest will affect estimated budget, therefore the costs risk will increase. Meanwhile, the variation of these variables will cause the market acceptance and operational expenses of project changed, increasing benefits risk. Some large and medium-sized projects are government-led investment projects, thus they have influenced by political factors largely. Changes in political will make construction of projects changed, so increasing the cost risk and design risk. 
In summary, a causal relationship diagram for risk analysis during project construction process to determine the state variables, auxiliary variables, input variables as well as the respective polarity between them is established, which is shown in Figure 1. Then we can make the corresponding flow diagram based on this causal relationship diagram, corresponding rate variables and other data, with a computer simulation conducting.

\section{Conclusions}

The good implementation of the project has great significance to the development of social economy. Analyzing project risk correctly and reasonably is important, but it is an intricate problem. Construction of projects involves various risk factors, and we can analyze various risks. More, it can also be more comprehensive with conventional methods, but only stay on a qualitative level, which cannot predict future trends.

A system dynamics model of the risk factor analysis during project construction is put forward, a causal relationship diagram is established, and the relationship between each variable initially is also identified. The causal relationship diagram is qualitative, which is influenced by subjective factors. Nevertheless, it provides a good starting point for further analysis. When the relevant equations and parameters being determined, we can simulate the model with Vensim software and obtain quantitative results.

\section{References}

[1] Lu, P. (2013) Research on Risks Management of Engineering Project. Science \& Technology Information, 17, 355-356.

[2] Li, H.G. (2013) Research on Risks Management Process of Engineering Project. Fujian Building Materials, 12 , 75-77.

[3] Zhu, Y.J. (2011) The Basis and Method for Engineering Project Risk Identification. Modern Enterprise, 9, $24-25$.

[4] Xu, G.Q. and Zou, J. (2006) The Method of System Dynamics: Principle, Characteristics and New Development. Journal of Harbin Institute of Technology (Social Sciences Edition), 8, 72-77.

[5] Forrester, J.W. (1985) Industrial Dynamics: A Breakthrough for Decision Makers. Harvard Business Review, 36, 3766.

[6] Forrester, J.W. (1968) Industrial Dynamics. Productivity Press, Cambridge.

[7] Rehan, R., Knight, M.A., Unger, A.J.A., et al. (2013) Development of a System Dynamics Model for Financially Sustainable Management of Municipal Watermain Networks. Water Research, 47, 7184-7205. http://dx.doi.org/10.1016/j.watres.2013.09.061

[8] Li, C.B. and Lu, G.S. (2012) System Engineering. Theory \& Practice, 32, 2731-2739.

[9] Sun, Y.C. and Sun, F.H. (2012) On Project Risk Characteristics and Prevention. Journal of Shenyang Jianzhu University (Social Science), 14, 386-388. 